\title{
Renin-Angiotensin System Blockade Is Associated with Exercise Capacity, Sympathetic Activity, and Endothelial Function in Patients with Chronic Kidney Disease
}

\author{
Jin Hee Jeong ${ }^{a, b}$ Justin D. Sprick ${ }^{a, b}$ Dana DaCosta ${ }^{a, b}$ Arshed A. Quyyumi ${ }^{c}$ \\ Jeanie Park ${ }^{a}, b$ \\ aDivision of Renal Medicine, Department of Medicine, Emory University School of Medicine, Atlanta, GA, USA; \\ ${ }^{b}$ Research Service Line, Department of Veterans Affairs Health Care System, Decatur, GA, USA; 'Division of \\ Cardiology, Department of Medicine, Emory University School of Medicine, Atlanta, GA, USA
}

\section{Keywords}

Renin-angiotensin system - Exercise capacity - Sympathetic activity - Endothelial dysfunction - Chronic kidney disease

\begin{abstract}
Background: Chronic kidney disease (CKD) patients have exercise intolerance and exaggerated blood pressure reactivity during exercise that are mediated by sympathetic nervous system (SNS) overactivation and decreased nitric oxide (NO) bioavailability. The activation of the renin-angiotensin system (RAS) increases SNS activation and reduces NO synthesis, and prior studies suggest that RAS blockade attenuates declines in physical function. We hypothesized that RAS inhibitor (RASi) use is associated with higher exercise capacity mediated by decreased SNS activity and increased NO-dependent endothelial function in CKD. Method: In 35 CKD patients ( $57 \pm 7$ years) and 20 controls (CONs) ( $53 \pm 8$ years), we measured exercise capacity (peak oxygen consumption $\left.\left[\mathrm{VO}_{2 \text { peak }}\right]\right)$, muscle sympathetic nervous activity (MSNA), and flow-mediated dilation (FMD) for NO-dependent endothelial function. Results: CKD patients treated with RASi (CKD + RASi, $n=25$ ) had greater $\mathrm{VO}_{2 \text { peak }}$ than CKD patients not treated with RASi (CKD no RASi, $n=10$ ), but lower $\mathrm{VO}_{2 \text { peak }}$ than
\end{abstract}

karger@karger.com www.karger.com/kbr

Karger $\stackrel{\text { ' }}{5}$

GOPEN ACCESS
(C) 2021 The Author(s)

Published by S. Karger AG, Basel

This is an Open Access article licensed under the Creative Commons Attribution-NonCommercial-4.0 International License (CC BY-NC) (http://www.karger.com/Services/OpenAccessLicense), applicable to the online version of the article only. Usage and distribution for commercial purposes requires written permission.
CONs ( $23.3 \pm 5.8$ vs. $16.4 \pm 2.9, p=0.007 ;$ vs. $30.0 \pm 7.7, p=$ $0.016 \mathrm{~mL} / \mathrm{min} / \mathrm{kg}$, respectively). CKD + RASi had lower resting MSNA and greater FMD than CKD no RASi. Compared to CONs, CKD + RASi had similar MSNA but lower FMD. $\mathrm{VO}_{2 \text { peak }}$ was positively associated with FMD $(r=0.417, p=0.038)$ and was predicted by the combination of FMD and RASi status $\left(r^{2}=0.344, p=0.01\right)$ and MSNA and RASi status $\left(r^{2}=0.575\right.$, $p=0.040$ ) in CKD patients. Conclusion: In summary, CKD patients with RASi have higher exercise capacity than those not on RASi. Higher exercise capacity in the RASi-treated group was associated with lower resting SNS activity and higher NO-dependent vascular endothelial function.

@ 2021 The Author(s)
Published by S. Karger AG, Basel

\section{Introduction}

Patients with chronic kidney disease (CKD) have decreased exercise capacity that begins in the early stages of disease development $[1,2]$. This exercise intolerance is associated with an increased risk of cardiovascular (CV) events, all-cause mortality [3-5], and progression of CKD $[6,7]$, and contributes to worsening of quality of life [8]. The causes of exercise intolerance in CKD are complex
Correspondence to:

Jeanie Park, jeanie.park@emory.edu 
and likely multifactorial due to a number of CKD-associated complications, including uremic myopathy, neuropathy, anemia, metabolic acidosis, chronic overactivation of the sympathetic nervous system (SNS), and endothelial dysfunction [9-11]. We have previously shown that CKD patients have abnormal hemodynamic and neurocirculatory responses during exercise, including an exaggerated increase in blood pressure (BP). These responses are mediated, in part, by SNS overactivation [12] and decreased endothelial nitric oxide (NO) bioavailability [13].

The renin-angiotensin system (RAS) plays an important role in various physiological processes including $\mathrm{BP}$ control and tissue perfusion, and RAS activation is implicated in the pathogenesis of hypertension (HTN) and CV target organ damage [14-16]. Pharmacological blockade of RAS activity through angiotensin-converting enzyme inhibitors (ACEis) and angiotensin receptor blockers (ARBs) is commonly used in CKD for BP control and proteinuria $[17,18]$, although not universally prescribed in all CKD patients, particularly in those without proteinuria [19]. Interestingly, in addition to CV and renal effects, evidence suggests that RAS may also modulate physical function declines in populations with physical functional impairment. For example, one observational study suggests that older adults treated with ACEis exhibited attenuation in age-related reductions in physical capacity compared to their peers not treated with ACEis [20]. Additionally, randomized controlled trials have revealed that ACEi or ARB treatment improves exercise capacity and exercise duration in older adults [21,22]. Beneficial effects of RAS blockade on physical function have also been documented in clinical populations including heart failure $[23,24]$ and peripheral artery disease [25]. However, the potential beneficial effects of RAS inhibition on physical function in CKD, on another patient population that is similarly characterized by exercise intolerance, have not been previously explored. Moreover, the mechanisms by which RAS blockade might mediate improvement in exercise capacity currently remain to be clarified.

RAS inhibition may positively impact exercise capacity through 2 distinct mechanisms: (1) modulation of SNS activity and (2) improvements in vascular endothelial function. CKD is characterized by both SNS overactivity at rest and during exercise $[12,26]$, as well as endothelial dysfunction that is linked to reduced exercise capacity $[10,13]$. RAS activation increases SNS activity [27, 28], stimulates the production of reactive oxygen species, and suppresses the synthesis of potent vasodilators such as $\mathrm{NO}$, which collectively promote endothelial dysfunction [29]. Decreased NO in turn also contributes to an in- creased central SNS output [30]. Thus, blockade of RAS by using RAS inhibitors (RASis) has been shown to ameliorate SNS hyperactivity $[26,31]$ and improve endothelial function $[32,33]$. Both of these may improve skeletal muscle blood flow regulation during exercise, thereby contributing to improvements in physical function. The purpose of this study was to investigate (1) the potential modulating effect of RASi on exercise capacity and (2) the potential physiological mechanisms of RASi effects on exercise capacity in patients with CKD. We hypothesized that the use of RASis is associated with greater exercise capacity in patients with $\mathrm{CKD}$, which is in part mediated by lower SNS activity and increased NO-dependent vascular endothelial function.

\section{Methods}

\section{Study Population}

Fifty-five sedentary participants including $35 \mathrm{CKD}$ patients and 20 age-matched controls (CONs) were recruited and enrolled from outpatient clinics at the Atlanta Veterans Affairs (VA) Healthcare System. All participants with CKD had a confirmed diagnosis of either stage 2 (estimated glomerular filtration rate [eGFR] of $60-89 \mathrm{~mL} \mathrm{~min}^{-1} 1.73 \mathrm{~m}^{-2}$ with a concomitant urinary microalbumin:creatinine ratio of $>30 \mathrm{mg} / \mathrm{g}$ ) or stage 3 (eGFR of 30-59 $\mathrm{mL} \mathrm{m^{-1 }} 1.73 \mathrm{~m}^{-2}$ ) CKD [34]. CKD participants had at least a 3 -month history of stable kidney function ( $\leq 10 \%$ fluctuation in the eGFR) and had comorbid HTN with a stable antihypertensive medication regimen before enrollment. Exclusion criteria included severe CKD (eGFR $\left.<30 \mathrm{~cm} \mathrm{~L}^{-1} \mathrm{~min}^{-1}\right)$; diabetes; HIV infection; heart failure; history of past coronary artery, cerebrovascular, aortic, or peripheral artery disease; symptomatic heart disease determined by electrocardiogram, stress test, and/or history; hepatic enzyme concentrations $>2$ times the upper limit of normal; severe anemia with a hemoglobin level $<10 \mathrm{~g} / \mathrm{dL}$; history of nephrolithiasis; any serious systemic disease that might influence survival; current treatment with clonidine; clinical BP $>160 / 90 \mathrm{~mm}$ $\mathrm{Hg}$ or $<110 / 60$; change in medications or surgery within the past 3 months; and drug or alcohol use disorders.

\section{Study Design}

After written informed consent was obtained, office BP, basic metabolic panel, and urinary albumin-to-creatinine ratio levels were obtained during a screening visit. During a separate visit, brachial artery flow-mediated dilation (FMD) was measured before maximal exercise testing. Muscle sympathetic nervous activity (MSNA) was obtained by microneurography during a separate visit. All measurements were obtained in a quiet, temperate $\left(21^{\circ} \mathrm{C}\right)$ environment, after abstaining from food, caffeine, smoking, and alcohol for at least $12 \mathrm{~h}$, and exercise for at least $24 \mathrm{~h}$. A standard snack of 2 Graham crackers and one small boxed juice was given after the FMD measurements and just before the exercise treadmill test. All participants reported having taken prescribed medications as normally directed. Patients with CKD were divided into dichotomous groups by RASi usage (either ACEi or ARB) at the time of the study visit. 
Measurements and Procedures

Blood Pressure

Baseline BP was measured after 5 min of rest in a seated position with the arm supported at the heart level using an appropriately sized cuff per American College of Cardiology/American Heart Association (ACC/AHA) guidelines [35] with an automated device (Omron, HEM-907XL; Omron Healthcare, Kyoto, Japan). The mean arterial BPs were calculated as $2 / 3$ diastolic BP (DBP) $+1 / 3$ systolic $\mathrm{BP}(\mathrm{SBP})$. Throughout the exercise treadmill test, $\mathrm{BP}$ was measured manually by a single investigator (J.P.). The heart rate (HR) was monitored using continuous ECG during the exercise treadmill test.

Maximal Exercise Treadmill Test

Participants underwent a modified Balke protocol, as previously described [5]. Briefly, participants were allowed to warm up for 2 min on a treadmill (GE T2100 controlled via GE Case V6.5 software) set to a speed of $1.5 \mathrm{mph}$ with the slope set to $0 \%$. At the start of the test, the speed was increased to $2.0 \mathrm{mph}$. At $3 \mathrm{~min}$, and every subsequent $3 \mathrm{~min}$, the treadmill slope was increased by $3.5 \%$. At the 18th minute, the speed was increased to $3.0 \mathrm{mph}$, while the treadmill slope was decreased to $12.5 \%$. The slope was again increased by $3.5 \%$ every 3 min until the participant achieved exhaustion. The HR was monitored continuously with ECG. BP was manually measured by the same investigator at $2 \mathrm{~min}$ and $30 \mathrm{~s}$ into each stage; the rate of perceived exertion was reported at $2 \mathrm{~min}$ and 50 s into each stage. Expired $\mathrm{O}_{2}, \mathrm{CO}_{2}$, and ventilation were recorded every 30 s (Sensormedics VMax Spectra 29) during exercise to determine exercise capacity (peak oxygen consumption $\left[\mathrm{VO}_{2 \text { peak }}\right]$ ). The $\mathrm{VO}_{2 \text { peak }}$ was defined as the highest $\mathrm{VO}_{2}$ observed during maximal exercise testing.

Muscle Sympathetic Nerve Activity

Microneurography was performed to record multiunit postganglionic MSNA directly from the peroneal nerve, as previously described [36]. A tungsten microelectrode (tip diameter 5-15 $\mu \mathrm{m}$ ) (Bioengineering, University of Iowa) was inserted into the peroneal nerve, and a reference microelectrode was inserted subcutaneously 1-2 cm from the recording electrode while the participants were in the supine position. The signals were amplified (total gain: $50,000-100,000)$, filtered $(700-2,000 \mathrm{~Hz})$, rectified, and integrated (time constant $0.1 \mathrm{~s}$ ) to obtain a mean voltage display of sympathetic nerve activity (Nerve Traffic Analyzer, model 662C-4; University of Iowa, Bioengineering) that was recorded by the LabChart 7 Program (PowerLab 16sp; ADInstruments). Continuous ECG was recorded simultaneously with the neurogram using a BioAmp System. Beat-to-beat arterial BP was measured via finger photoplethysmography [37]. Absolute values of BP were internally calibrated using a concomitant upper arm BP reading and were calibrated at the start and every 15 min throughout the study. The tungsten microelectrode was manipulated to obtain a satisfactory nerve recording that met previously established criteria [38]. After 10 min of rest, baseline $\mathrm{BP}, \mathrm{HR}$, respiratory rate, and MSNA were recorded continuously for $10 \mathrm{~min}$.

\section{Brachial Artery FMD}

A forearm occlusion cuff was placed on participants while they were in a supine position. A $13-\mathrm{MHz}$ high-resolution ultrasound transducer (Acuson Aspen) was placed longitudinally 2-10 cm above the antecubital fossa to record brachial artery measurements.
Baseline values were obtained by averaging the diameter and blood velocity over 3 cardiac cycles measured via ECG gating to capture end-diastolic arterial diameters. The forearm cuff was inflated to suprasystolic levels (50 $\mathrm{mm} \mathrm{Hg}$ above SBP) using a rapid cuff inflator (D.E. Hokanson) for $5 \mathrm{~min}$. The peak hyperemic blood velocity was measured by Doppler ultrasound during the first $10 \mathrm{~s}$ following cuff release. Diameter measurements were obtained 60 and $90 \mathrm{~s}$ following cuff release. FMD calculations were made using 60 -s and 90 -s measurements to determine the peak diameter. Arterial diameters were measured and analyzed by a single investigator blinded to clinical status of the participant from digitized images utilizing customized software (Medical Imaging Applications). FMD is given as the per cent change in the artery diameter from baseline: (peak hyperemic diameter - baseline diameter)/baseline diameter. The shear rate at baseline and peak hyperemia was calculated as $4 \times$ peak blood velocity/arterial diameter. FMD values were then normalized for the peak hyperemic shear rate. This calculation of the shear rate is consistent with our previous reports [39].

\section{Data Analysis}

Muscle Sympathetic Nerve Activity

MSNA and ECG data were exported and analyzed offline via specialized software (WinCPRS; Absolute Aliens, Turku, Finland). $\mathrm{R}$-waves were detected and marked from the continuous ECG recording. MSNA bursts were automatically detected by the program using the following criteria: 3:1 burst-to-noise ratio within a 0.5 -s search window, with an average latency in burst occurrence of 1.2-1.4 s from the previous R-wave. After automatic detection, the ECG and MSNA neurograms were visually inspected for accuracy of detection by a single investigator (J. Park). MSNA was expressed as the burst frequency (bursts/min) and burst incidence (bursts/100 heartbeats).

\section{Statistics}

Values are presented as mean \pm standard deviation unless otherwise noted. Participant characteristics and primary outcomes $\left(\mathrm{VO}_{2 \text { peak }}, \mathrm{MSNA}\right.$, and FMD) between RASi users and non-RASi users in CKD patients were compared between groups via independent $t$ tests for continuous variables, $\chi^{2}$ tests for categorical variables, and ANOVA for adjusted comparison analysis with $\mathrm{VO}_{2 \text { peak }}$ in exercise hemodynamic variables. The comparison between CKD and CON groups were also performed via independent $t$ tests. The Pearson correlation test was performed to examine the linear relationship between $\mathrm{VO}_{2 \text { peak }}$ and MSNA and FMD in CKD patients. Multivariable linear regression was used to describe the predictability of MSNA and FMD combined with and without RASi usage on $\mathrm{VO}_{2 \text { peak }}$ in $\mathrm{CKD}$ patients. An $\alpha<0.05$ was considered statistically significant for all analyses. All analyses were performed using SPSS version 26.0 (IBM Corporation, Somers, NY, USA).

\section{Results}

\section{Participant Characteristics}

A total of 55 participants including patients with CKD $(n=35)$ and age-matched CONs without CKD $(n=20)$ completed the study. The CKD group was further divided by RASi usage: CKD no RASi $(N=10)$ and CKD + RASi 
Table 1. Participant characteristics

\begin{tabular}{|c|c|c|c|c|c|}
\hline Characteristics & $\begin{array}{l}\text { CKD no RASi } \\
(n=10)\end{array}$ & $\begin{array}{l}\text { CKD + RASi } \\
(n=25)\end{array}$ & $p$ value ${ }^{\#}$ & $\begin{array}{l}\text { CON } \\
(n=20)\end{array}$ & $p$ value $e^{\&}$ \\
\hline Age, years & $56.6 \pm 6.3$ & $56.8 \pm 7.4$ & 0.911 & $52.5 \pm 8.2$ & 0.065 \\
\hline Sex (male), $n(\%)$ & $10(100)$ & $25(100)$ & 0.226 & $21(95)$ & 0.375 \\
\hline \multicolumn{6}{|l|}{ Race, $n(\%)$} \\
\hline Black & $10(100)$ & $23(92)$ & 0.504 & $19(86)$ & 0.248 \\
\hline White & 0 & $2(8)$ & & $3(14)$ & \\
\hline Height, cm & $180.0 \pm 4.9$ & $180.8 \pm 7.0$ & 0.787 & $179.7 \pm 6.4$ & 0.724 \\
\hline $\mathrm{BMI}, \mathrm{kg} / \mathrm{m}^{2}$ & $26.0 \pm 14.2$ & $32.6 \pm 8.0$ & 0.095 & $28.8 \pm 4.5$ & 0.422 \\
\hline $\mathrm{HTN}, n(\%)$ & $10(100)$ & $25(100)$ & & $12(60)$ & 0.000 \\
\hline \multicolumn{6}{|l|}{ Antihypertensive medications, $n$ (\%) } \\
\hline ACEi/ARB & 0 & $17 / 8(100)$ & 1.000 & $3(17)$ & 0.002 \\
\hline $\mathrm{CCB}$ & $7(70)$ & $14(56)$ & 0.458 & $6(27)$ & 0.015 \\
\hline Diuretics & $3(30)$ & $11(44)$ & 0.698 & $9(41)$ & 0.598 \\
\hline$\beta$-Blockers & $5(50)$ & $7(28)$ & 0.380 & $1(5)$ & 0.001 \\
\hline Aldosterone receptor blockers & $3(30)$ & 0 & 0.015 & $1(13)$ & 0.653 \\
\hline a-Blockers & 0 & $4(16)$ & 0.554 & $1(13)$ & 0.519 \\
\hline Hydralazine statin & $3(30)$ & 0 & 0.020 & 0 & 0.271 \\
\hline eGFR, $\mathrm{mL} \min ^{-1} 1.73 \mathrm{~m}^{-2}$ & $51.4 \pm 19.1$ & $48.3 \pm 10.8$ & 0.540 & $89.5 \pm 12.6$ & 0.000 \\
\hline UACR, $\mathrm{mg} / \mathrm{g}$ & $93 \pm 122$ & $147 \pm 236$ & 0.629 & $1 \pm 0$ & 0.006 \\
\hline
\end{tabular}

Values are expressed as means $\pm \mathrm{SD}$. RASi, renin-angiotensin system inhibitors; CKD, chronic kidney disease; CONs, controls; BMI, body mass index; CCB, calcium channel blocker; $A C E$, angiotensin-converting enzyme inhibitor; ARB, angiotensin receptor blocker; eGFR, estimated glomerular filtration rate; UACR, urinary albumin-to-creatinine ratio; HTN, hypertension; SD, standard deviation. " Indicates group comparison by RASi usage in CKD patients. \& Indicates group comparison between all CKD patients versus CON participants.

Table 2. Exercise hemodynamic parameters in CKD patients with and without RASi and CONs

\begin{tabular}{|c|c|c|c|c|c|}
\hline Hemodynamics & $\begin{array}{l}\text { CKD no RASi } \\
(n=7)\end{array}$ & $\begin{array}{l}\text { CKD + RASi } \\
(n=18)\end{array}$ & $p$ value $\#$ & $\begin{array}{l}\text { CON } \\
(n=20)\end{array}$ & $p$ value $\&$ \\
\hline SBP pre, mm Hg & $137 \pm 9$ & $132 \pm 14$ & 0.329 & $124 \pm 13$ & 0.018 \\
\hline DBP pre, $\mathrm{mm} \mathrm{Hg}$ & $84 \pm 4$ & $84 \pm 10$ & 0.853 & $75 \pm 9$ & 0.003 \\
\hline MAP pre, $\mathrm{mm} \mathrm{Hg}$ & $102 \pm 5$ & $99 \pm 10$ & 0.543 & $91 \pm 9$ & 0.002 \\
\hline HR pre, bpm & $63 \pm 11$ & $64 \pm 12$ & 0.965 & $67 \pm 15$ & 0.332 \\
\hline SBP max, mm Hg & $174 \pm 27$ & $191 \pm 28$ & 0.181 & $183 \pm 23$ & 0.719 \\
\hline DBP max, mm Hg & $88 \pm 5$ & $89 \pm 12$ & 0.764 & $84 \pm 10$ & 0.118 \\
\hline MAP max, mm Hg & $115 \pm 11$ & $121 \pm 15$ & 0.374 & $114 \pm 14$ & 0.232 \\
\hline HR max, bpm & $114 \pm 25$ & $139 \pm 25$ & 0.033 & $150 \pm 17$ & 0.012 \\
\hline SBP 4 min-post, mm Hg & $136 \pm 5$ & $133 \pm 13$ & 0.611 & $130 \pm 16$ & 0.540 \\
\hline DBP 4 min-post, mm Hg & $85 \pm 6$ & $80 \pm 9$ & 0.261 & $74 \pm 13$ & 0.036 \\
\hline MAP 4 min-post, $\mathrm{mm} \mathrm{Hg}$ & $102 \pm 4$ & $98 \pm 9$ & 0.284 & $93 \pm 11$ & 0.053 \\
\hline HR 4 min-post, bpm & $76 \pm 5$ & $90 \pm 18$ & 0.108 & $96 \pm 15$ & 0.066 \\
\hline
\end{tabular}

Values are expressed as mean \pm SD. Significant $p$ values are shown in bold. RASi, renin-angiotensin system inhibitors; CKD, chronic kidney disease; CONs, controls; $\mathrm{HEA}$, healthy control; $\mathrm{VO}_{2 \text { peak, }}$ peak oxygen consumption; $\mathrm{SBP}$, systolic blood pressure; DBP, diastolic blood pressure; MAP, mean arterial blood pressure; HR, heart rate; pre, measurement obtained just prior to the start of the exercise; max, the maximal value obtained during the exercise; 4 min-post, measurement obtained 4 min after exercise cessation; SD, standard deviation. \# Indicates group comparison by RASi usage in CKD patients. ${ }^{\&}$ Indicates group comparison between all CKD patients versus CON participants. 
A

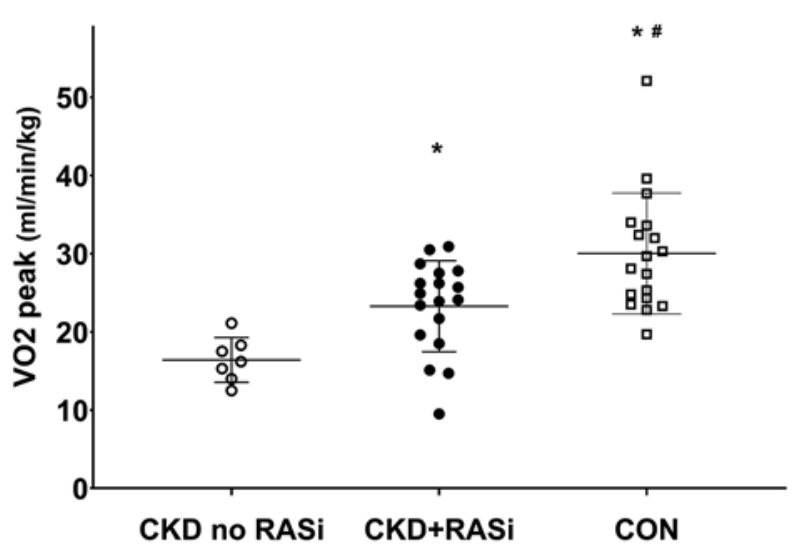

C

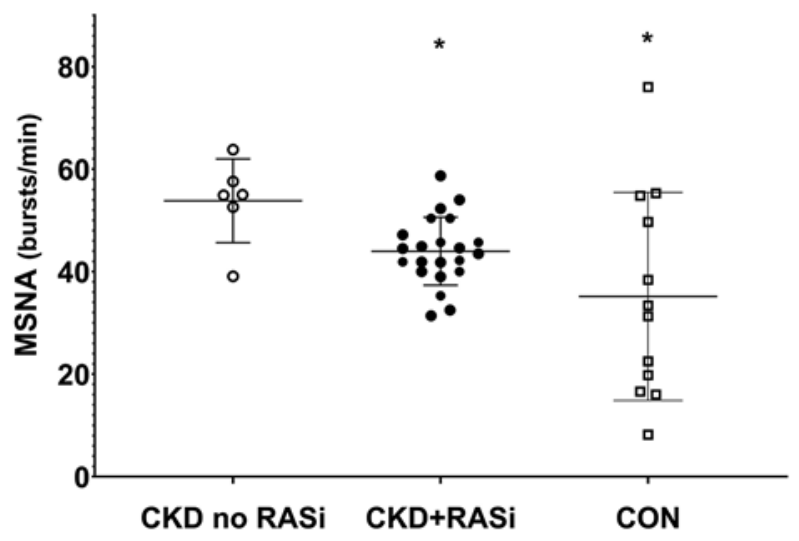

D
B
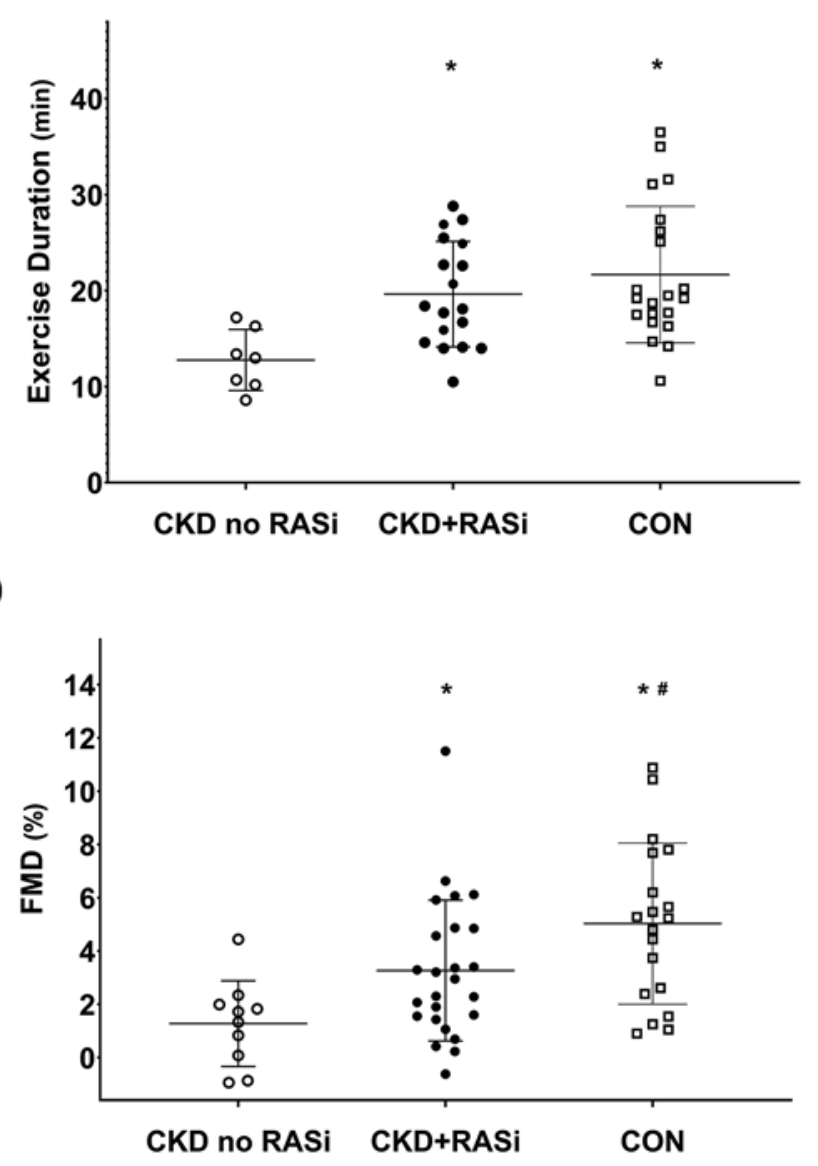

Fig. 1. $\mathrm{VO}_{2 \text { peak }}(\mathbf{A})$, exercise duration in the maximal treadmill test (B), MSNA (C), and FMD (D) in CKD patients treated with RASis $(\mathrm{CKD}+\mathrm{RASi})$ and without RASi (CKD no RASi) and CONs without CKD. *Indicates a significant difference $(p<0.05)$ from the CKD no RASi group. "Indicates a significant difference $(p<0.05)$

$(N=25)$. Participant characteristics are shown in Table 1. The majority of participants in all groups were Black males. In patients with CKD, the main causes of CKD were $\operatorname{HTN}(n=14)$, an unknown cause combined with HTN $(n=8)$, polycystic kidney disease $(n=2)$, glomerulonephritis $(n=1)$, and unknown $(n=10)$. All participants with CKD and $60 \%$ of CONs had HTN. None of the participants had comorbid diabetes (exclusion criterion). There were no significant differences in demographics, anthropometric characteristics, antihypertensive medication, eGFR, urinary albumin-to-creatinine ratio, or HMG CoA reductase inhibitors (statins) use between CKD no RASi and CKD + RASi. Compared to CONs, from the CKD + RASi group. CKD, chronic kidney disease; RASi, renin-angiotensin system inhibitor; MSNA, muscle sympathetic nervous activity; FMD, flow-mediated dilation; CON, control; $\mathrm{VO}_{2 \text { peak, }}$, peak oxygen consumption.

CKD patients as a whole had greater proportion of hypertensives and higher rsates of antihypertensive medication usage (calcium channel blocker, RASi, and $\beta$-blockers) $(p<0.05$ for all).

\section{Exercise Hemodynamics in CKD Patients with and} without RASi Treatment

Hemodynamics before (pre), during ( $\max )$, and after (post) maximal treadmill exercise testing for all groups are shown in Table 2. There was no difference in hemodynamic measures at pre- and postexercise between CKD no RASi and CKD + RASi. Compared to CKD groups, CONs had lower SBP, DBP, and mean arterial BP but not 


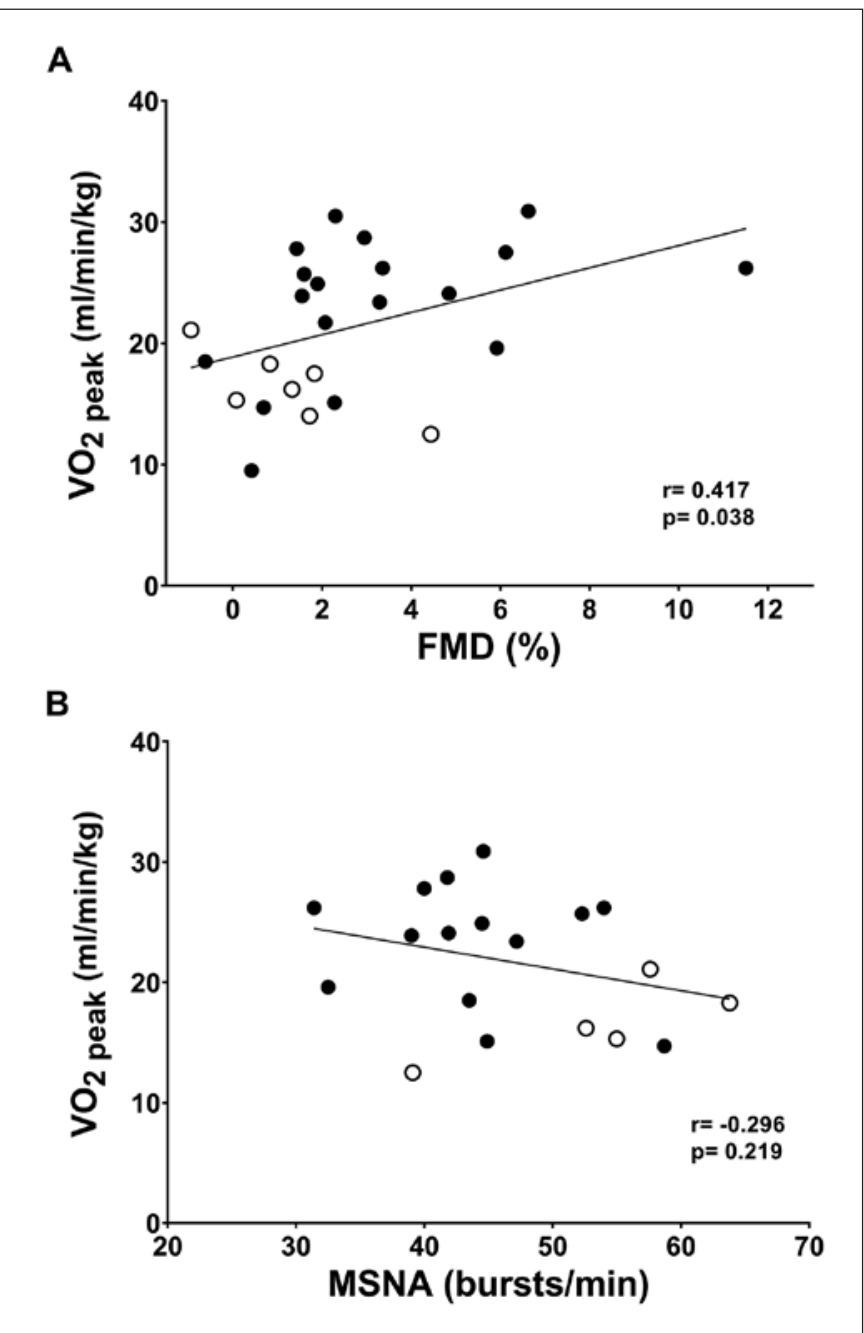

Fig. 2. Association between $\mathrm{VO}_{2 \text { peak }}$ and $\mathrm{FMD}(\mathbf{A})$ and MSNA (B) in patients with CKD. Closed circles $(\bullet)$ for CKD patients with RASi and open circles $(\mathrm{O})$ for CKD patients without RASi. CKD, chronic kidney disease; RASi, renin-angiotensin system inhibitor; MSNA, muscle sympathetic nervous activity; FMD, flow-mediated dilation; $\mathrm{VO}_{2 \text { peak }}$, peak oxygen consumption.

HR at pre-exercise, lower DBP at postexercise, and higher max HR during exercise. CKD + RASi had a greater max HR during maximal treadmill exercise than CKD no RASi, which was no longer significant after adjusting for $\mathrm{VO}_{2 \text { peak }}$ levels or exercise duration ( $p>0.05$ for all).

Exercise Capacity, Sympathetic Nervous Activity, and Endothelial Function in CKD Patients with and without RASi Treatment

$\mathrm{CKD}+\mathrm{RASi}$ had higher $\mathrm{VO}_{2 \text { peak }}$ than CKD no RASi but lower $\mathrm{VO}_{2 \text { peak }}$ than CONs $(23.3 \pm 5.8$ vs. $16.4 \pm 2.9$ $\mathrm{mL} / \mathrm{min} / \mathrm{kg} ; p=0.007$, vs. $30.0 \pm 7.7 ; p=0.016 \mathrm{~mL} / \mathrm{min} /$ kg, respectively; Fig. 1A), suggesting that RASi use is associated with higher exercise capacity in CKD but is not associated with restoration of exercise capacity to the level of CONs. CKD + RASi had longer exercise duration during maximal treadmill exercise testing than SCKD no $\mathrm{RASi}$, while there was no difference in exercise duration between CKD + RASi and CONs (17 \pm 5 vs. $12 \pm 5 \mathrm{~min}$; $p=0.005$, vs. $22 \pm 7$ min; $p=0.819$, respectively; Fig. $1 \mathrm{~B}$ ), suggesting that RASi use is associated with increased endurance in CKD to the level of CONs.

CKD + RASi had lower resting MSNA than CKD no RASi but similar resting MSNA levels compared to CONs $(43.9 \pm 7.1$ vs. $53.8 \pm 8.2$ bursts $/ \mathrm{min} ; p=0.009$, vs. $38.0 \pm 20.3$ bursts $/ \mathrm{min} ; p=0.103$, respectively; Fig. 1C), suggesting that RASi use is associated with sympathoinhibition in CKD to the level of CONs. CKD + RASi had higher brachial artery FMD than CKD no RASi but lower FMD than CONs $(3.2 \pm 2.6$ vs. $1.3 \pm$ $1.6 \% ; p=0.007$, vs. $5.0 \pm 3.0 \% ; p=0.045 \%$, respectively; Fig. 1D), suggesting that RASi use is associated with higher NO-mediated endothelial function in CKD but is not associated with restoration of endothelial function to the level of CONs.

\section{Predictors of Exercise Capacity in CKD Patients}

$\mathrm{VO}_{2 \text { peak }}$ was positively associated with $\mathrm{FMD}(r=0.417$, $p=0.038)$ but was not associated with resting MSNA ( $r=$ $-0.296, p=0.219$ ) in CKD patients (Fig. 2A, B, respectively). Multivariable linear regression analysis demonstrated that both FMD alone and combined with RASi usage status significantly predicts $\mathrm{VO}_{2 \text { peak }}$ in CKD patients (Table 3, Model 1,2). Although MSNA alone was not predictive, the combination of MSNA and RASi usage significantly predicts $\mathrm{VO}_{2 \text { peak }}$ in CKD patients (Table 3, Model 3, 4). When FMD, MSNA, and RASi were considered together as potential predictors, there was a trend toward predicting $\mathrm{VO}_{2 \text { peak }}$ in $\mathrm{CKD}$ patients (Table 3, Model 5).

\section{Discussion}

The present study demonstrates that CKD patients on RASis have higher exercise capacity than a similar cohort of CKD patients not on RASis. Greater exercise capacity in RASi-treated group was associated with lower resting SNS activity and higher NO-dependent vascular endothelial function in patients with CKD. These findings support our hypothesis that the RASi is associated with improved exercise capacity in $\mathrm{CKD}$, and these effects may 
Table 3. Multivariable regression analysis with MSNA, FMD, and RASi usage for $\mathrm{VO}_{2 \text { peak }}$ in $C K D$ patients

\begin{tabular}{|c|c|c|c|c|c|c|c|}
\hline Model & $\begin{array}{l}\text { Dependent } \\
\text { variable }\end{array}$ & Predictor & $\begin{array}{l}\text { Unstandardized } \\
\beta\end{array}$ & $\begin{array}{l}\text { Standardized } \\
\beta\end{array}$ & $R^{2}$ & $\operatorname{Adj} R^{2}$ & $\begin{array}{l}\text { Model } \\
p \text { value }\end{array}$ \\
\hline 1 & $\mathrm{VO}_{2 \text { peak }}$ & FMD & 0.919 & 0.414 & 0.174 & 0.138 & 0.038 \\
\hline 2 & $\mathrm{VO}_{2 \text { peak }}$ & $\begin{array}{l}\text { FMD } \\
\text { RASi }\end{array}$ & $\begin{array}{l}0.609 \\
5.696\end{array}$ & $\begin{array}{l}0.276 \\
0.436\end{array}$ & 0.344 & 0.284 & 0.010 \\
\hline 3 & $\mathrm{VO}_{2 \text { peak }}$ & MSNA & -0.181 & -0.296 & 0.088 & 0.034 & 0.219 \\
\hline 4 & $\mathrm{VO}_{2 \text { peak }}$ & $\begin{array}{l}\text { MSNA } \\
\text { RASi }\end{array}$ & $\begin{array}{l}-0.011 \\
6.762\end{array}$ & $\begin{array}{l}-0.018 \\
0.566\end{array}$ & 0.575 & 0.247 & 0.040 \\
\hline 5 & $\mathrm{VO}_{2 \text { peak }}$ & $\begin{array}{l}\text { FMD } \\
\text { MSNA } \\
\text { RASi }\end{array}$ & $\begin{array}{l}0.535 \\
0.104 \\
6.672\end{array}$ & $\begin{array}{l}0.293 \\
0.170 \\
0.558\end{array}$ & 0.379 & 0.255 & 0.061 \\
\hline
\end{tabular}

Significant $p$ values are shown in bold. MSNA, muscle sympathetic nervous activity; FMD, flow-mediated dilation; RASi, renin-angiotensin system inhibitors; $\mathrm{VO}_{2 \text { peak }}$ peak oxygen consumption; $\mathrm{CKD}$, chronic kidney disease.

be mediated by via differences in SNS activity and NOdependent endothelial function.

Exercise intolerance is a clinical hallmark of CKD that begins in the early stages of CKD [1]. Reduced exercise capacity is an independent predictor of the development and progression of CKD $[6,7]$ and is associated with increased risk of hospitalization and mortality [3-5]. This impairment in physical performance may also result in a reduction in exercise capacity as well as an inability to perform activities of daily living, therefore negatively affecting quality of life in patients with $\mathrm{CKD}$ [8]. The mechanisms of exercise intolerance in CKD are complex, involving central and peripheral abnormalities that limit efficient oxygen delivery and utilization during exercise [9-11]. The present study adds novel evidence that pharmacological inhibition of the RAS is associated with beneficial effects on exercise capacity in patients with CKD. Previous studies suggest that RAS blockade may prevent or restore declines in physical functioning in populations with low physical functioning including older adults [40] and patients with heart failure [23, 24]. ACEi treatment was associated with an attenuated age-related reduction in the walking speed and muscle strength in 641 hypertensive elderly women over the course of 3 years [20]. Moreover, a randomized controlled trial demonstrated that ACEi treatment improved physical function in functionally impaired elderly individuals, and the degree of improvement was equivalent to that reported after 6 months of exercise training [21]. Likewise, ARB treatment also showed an improvement in physical function in the elderly [22], peripheral artery disease [25], and in animal models [18] and patients with heart failure [23, 24]. Conversely, other studies [41-44] have reported no association between RASi use and indicators of physical function in functionally impaired populations. Self-reported incidence of frailty was similar between ACEi users and nonusers regardless of the duration of ACEi usage in older women [44]. RASi use failed to improve exercise capacity in older adults with high CV risk [41] and individuals with myocardial hypertrophy [43] and right-ventricular dysfunction [42]. These mixed results suggest that the interaction between RASi and exercise performance cannot be generalized in heterogenous populations characterized by exercise intolerance, likely due to different origins of physical functional impairment (central cardiac, peripheral limitations, or a mixture of both) and different mechanisms of population-specific RASidriven health benefits.

We observed a positive effect of RASi on exercise capacity in a population of predominantly Black males with mild-to-moderate CKD. The mechanisms by which RAS inhibition may modulate physical functioning remain poorly understood. RASis are widely used in the treatment of HTN and CV diseases due to their BP-lowering effects and end-organ protection [14-16]. In CKD, RASi treatment has been shown to exert reno-protective and antiproteinuric effects beyond their well-known antihypertensive effects and is thus recommended as a first-line antihypertensive agent in this population $[17,45]$. However, not all CKD patients, particularly those without proteinuria, are necessarily treated with RASi since the greatest nephro-protective effects of RASi are noted in 
patients with proteinuria [45]. The CV-protective effects of RASi are mediated primarily through inhibition of the production and activity of the main downstream effector angiotensin II (Ang II) and its interaction with angiotensin type 1 receptor $\left(\mathrm{AT}_{1} \mathrm{R}\right)$ [46]. Inhibition of Ang II$A T_{1} R$ signaling either by ACEis or ARBs as well as suppression of the degradation of bradykinin by ACEis promotes NO production [29], leading to improved endothelial function $[32,33]$. This improvement in endothelial function with a RASi and the resultant improvements in blood flow may therefore be associated with improvements in physical function by optimizing nutrient and oxygen supply to exercising skeletal muscle [47]. In this regard, improvement in insulin sensitivity, glycogen storage, and glucose uptake by skeletal muscles have been reported with RASi treatment, supporting a role in improved muscle metabolic efficiency [48]. Our results demonstrate that despite similar resting $\mathrm{BP}$ between CKD patients treated with RASi and those not treated with RASi, the RASi-treated group exhibited greater endothelial function, which was associated with increased exercise capacity in CKD. These results are consistent with previous findings that similarly report improvements in endothelial function following RASi treatment $[32,33]$.

Ang II is also known to enhance SNS activation both peripherally and centrally by facilitating presynaptic release of norepinephrine (NE) and inhibiting NE reuptake at sympathetic nerve terminals, enhancing the density of sympathetic innervation and binding to $\mathrm{AT}_{1} \mathrm{R}$ in SNS control centers in the brain $[27,28]$. CKD is characterized by chronically elevated SNS activity, and RASi treatment has been shown to reduce MSNA levels in patients with CKD [26, 31], suggesting a RAS-dependent sympathetic hyperactivity in CKD. Ang II-mediated increases in NE release could contribute to a reduction in physical capacity by impairing blood flow and oxygen delivery to exercising skeletal muscle. Attenuation of sympathetic vasoconstriction in exercising skeletal muscles, termed functional sympatholysis, is a protective physiological mechanism to ensure proper muscle perfusion to meet increased metabolic demands during exercise [49]. Functional sympatholysis has been shown to be impaired in patient populations characterized by exaggerated SNS activity such as HTN [50] and end-stage kidney disease patients [51]. Functional sympatholysis can be restored with ARBs but not with other classes of antihypertensives in patients with HTN [50, 52], suggesting an important role of RAS in sympathetically mediated vasoconstriction in exercising skeletal muscles. Although the underlying mechanisms are unknown, our findings support the contention that RASi-mediated reduction in SNS activity may play a role in improved exercise capacity in CKD since hypertensive CKD patients on a RASi had a lower resting MSNA level, which was in turn associated with and predictive of increased exercise capacity in multivariate analyses. Interestingly, the predictability of MSNA for exercise capacity was enhanced when RASi usage was considered as a co-predictor suggesting a potential synergic effect of SNS and RAS inhibition in preserving exercise capacity in CKD. However, care should be taken in the interpretation of these cross-sectional analyses, and future long-term studies are needed to examine the effects of RASi treatment on sympathetic vasoconstriction and its potential contribution to exercise capacity in patients with CKD.

\section{Limitations}

This study was an observational study; therefore, the causal relationship between RASi usage, exercise capacity, SNS activity, and endothelial function cannot be determined in this cross-sectional study. This study is also limited by a small sample size, and longitudinal studies with larger sample sizes are needed to fully ascertain these findings of relationship between RAS blockade, SNS activity, and endothelial function. Only males were enrolled, and thus, the present findings may not be generalizable to females. Furthermore, only mild-to-moderate CKD (stages II and III) were included; therefore, the results may not be generalizable to more severe CKD (stage IV) or end-stage kidney disease. The RASi group combined patients who were on either ACEis or ARBs and was not powered to detect differences between ACEi and ARB subgroups. The dosage and duration of RASi administration and other concomitant vasoactive and chronotropic mediations were not controlled; however, there was no significant difference in antihypertensive medication usage between groups with and without RASi. Functional sympatholysis was not measured, so it is unclear if reductions in SNS activity improve exercise capacity in CKD by improving muscle perfusion.

\section{Conclusion}

Our results indicate that hypertensive CKD patients treated with a RASi have higher exercise capacity that is associated with lower SNS activity and higher endothelial function. These findings provide novel insights on the 
potential beneficial effects of RAS blockade on physical function in CKD and the potential mechanistic roles of SNS activity and endothelial function on RAS-mediated preservation of exercise capacity, although care should be taken with the interpretation due to the small sample size and the cross-sectional design of the study. Long-term randomized trials with a larger sample size are needed to examine causal links between RASi treatment and physical function in CKD, as well as the underlying autonomic and vascular mechanisms.

\section{Statement of Ethics}

This study was conducted ethically in accordance with World Medical Association Declaration of Helsinki. This study was approved by the Emory University Institutional Review Board and the Atlanta VA Healthcare System Research and Development Committee (IRB00019181). Written informed consent was obtained from all participants to participate in the study.

\section{Conflict of Interest Statement}

No conflicts of interest, financial or otherwise, are declared by the author(s).

\section{Funding Sources}

This work was supported by the National Institutes of Health (NIH) R01 HL135183; NIH R61 AT10457; NIH Training Grant T32 DK00756; and NIH F32HL147547; Merit Review Award number I01CX001065 from the United States Department of VA Clinical Sciences Research and Development Program; Department of VA, Veterans Health Administration, Office of Research and Development and the Clinical Studies Center of the Atlanta VA Health Care System, Decatur, GA; and the Foundation for Atlanta Veterans Education and Research (FAVER).

\section{Author Contributions}

J.H.J. and J.P. conceived and designed the research; D.D., A.A.Q., and J.P. performed the experiments; J.H.J., J.D.S., and J.P. analyzed the data; J.H.J., J.D.S., A.A.Q., and J.P. interpreted the results of the experiments; J.H.J. and J.P. prepared the figures; J.H.J. and J.P. drafted the manuscript; J.H.J., J.D.S., A.A.Q., D.D., and J.P. edited and revised the manuscript; J.H.J., J.D.S., A.A.Q., D.D., and J.P. approved the final version of the manuscript.

\section{Data Availability Statement}

All data generated or analyzed during this study are included in this article. Further inquiries can be directed to the corresponding author.

\section{References}

1 Howden EJ, Weston K, Leano R, Sharman JE, Marwick TH, Isbel NM, et al. Cardiorespiratory fitness and cardiovascular burden in chronic kidney disease. J Sci Med Sport. 2015; 18(4):492-7.

2 Wallin H, Asp AM, Wallquist C, Jansson E, Caidahl K, Hylander Rössner B, et al. Gradual reduction in exercise capacity in chronic kidney disease is associated with systemic oxygen delivery factors. PLoS One. 2018;13(12): e0209325.

3 Morishita S, Tsubaki A, Shirai N. Physical function was related to mortality in patients with chronic kidney disease and dialysis. Hemodial Int. 2017;21(4):483-9.

4 Ting SM, Iqbal H, Kanji H, Hamborg T, Aldridge N, Krishnan N, et al. Functional cardiovascular reserve predicts survival pre-kidney and post-kidney transplantation. J Am Soc Nephrol. 2014;25(1):187-95.

5 Sietsema KE, Amato A, Adler SG, Brass EP. Exercise capacity as a predictor of survival among ambulatory patients with end-stage renal disease. Kidney Int. 2004;65(2):719-24.

6 Kokkinos P, Faselis C, Myers J, Sui X, Zhang J, Tsimploulis A, et al. Exercise capacity and risk of chronic kidney disease in US veterans: a cohort study. Mayo Clin Proc. 2015;90(4): $461-8$.
7 Chang YT, Wu HL, Guo HR, Cheng YY, Tseng CC, Wang MC, et al. Handgrip strength is an independent predictor of renal outcomes in patients with chronic kidney diseases. Nephrol Dial Transplant. 2011;26(11):3588-95.

8 Lopes AA, Lantz B, Morgenstern H, Wang M, Bieber BA, Gillespie BW, et al. Associations of self-reported physical activity types and levels with quality of life, depression symptoms, and mortality in hemodialysis patients: the DOPPS. Clin J Am Soc Nephrol. 2014;9(10): 1702-12.

9 Kosmadakis GC, Bevington A, Smith AC, Clapp EL, Viana JL, Bishop NC, et al. Physical exercise in patients with severe kidney disease. Nephron Clin Pract. 2010;115(1):c7-16.

10 Van Craenenbroeck AH, Van Craenenbroeck EM, Van Ackeren K, Hoymans VY, Verpooten GA, Vrints CJ, et al. Impaired vascular function contributes to exercise intolerance in chronic kidney disease. Nephrol Dial Transplant. 2016;31(12):2064-72.

11 Nishikawa M, Ishimori N, Takada S, Saito A, Kadoguchi T, Furihata T, et al. AST-120 ameliorates lowered exercise capacity and mitochondrial biogenesis in the skeletal muscle from mice with chronic kidney disease via reducing oxidative stress. Nephrol Dial Transplant. 2015;30(6):934-42.
12 Park J, Quyyumi AA, Middlekauff HR. Exercise pressor response and arterial baroreflex unloading during exercise in chronic kidney disease. J Appl Physiol. 2013;114(5):538-49.

13 Downey RM, Liao P, Millson EC, Quyyumi AA, Sher S, Park J. Endothelial dysfunction correlates with exaggerated exercise pressor response during whole body maximal exercise in chronic kidney disease. Am J Physiol Renal Physiol. 2017;312(5):F917-24.

14 Mancia G, Fagard R, Narkiewicz K, Redón J, Zanchetti A, Böhm M, et al. 2013 ESH/ESC guidelines for the management of arterial hypertension: the Task Force for the Management of Arterial Hypertension of the European Society of Hypertension (ESH) and of the European Society of Cardiology (ESC). Eur Heart J. 2013;34(28):2159-219.

15 Heart Outcomes Prevention Evaluation Study Investigators; Yusuf S, Sleight P, Pogue J, Bosch J, Davies R, et al. Effects of an angiotensin-converting-enzyme inhibitor, ramipril, on cardiovascular events in high-risk patients. N Engl J Med. 2000;342(3):145-53.

16 Brenner BM, Cooper ME, de Zeeuw D, Keane WF, Mitch WE, Parving HH, et al. Effects of losartan on renal and cardiovascular outcomes in patients with type 2 diabetes and nephropathy. N Engl J Med. 2001;345(12):861-9. 
17 Cheung AK, Chang TI, Cushman WC, Furth SL, Ix JH, Pecoits-Filho R, et al. Blood pressure in chronic kidney disease: conclusions from a kidney disease: Improving Global Outcomes (KDIGO) Controversies Conference. Kidney Int. 2019;95(5):1027-36.

18 Carter CS, Giovannini S, Seo DO, DuPree J, Morgan D, Chung HY, et al. Differential effects of enalapril and losartan on body composition and indices of muscle quality in aged male Fischer 344 x Brown Norway rats. Age. 2011;33(2):167-83.

19 Weir MR, Lakkis JI, Jaar B, Rocco MV, Choi MJ, Kramer HJ, et al. Use of renin-angiotensin system blockade in advanced CKD: an NKF-KDOQI controversies report. Am J Kidney Dis. 2018;72(6):873-84.

20 Onder G, Penninx BW, Balkrishnan R, Fried LP, Chaves PH, Williamson J, et al. Relation between use of angiotensin-converting enzyme inhibitors and muscle strength and physical function in older women: an observational study. Lancet. 2002;359(9310):92630.

21 Sumukadas D, Witham MD, Struthers AD, McMurdo ME. Effect of perindopril on physical function in elderly people with functional impairment: a randomized controlled trial. CMAJ. 2007;177(8):867-74.

22 Simpson KL, McClellan KJ. Losartan: a review of its use, with special focus on elderly patients. Drugs Aging. 2000;16(3):227-50.

23 Warner JG Jr, Metzger DC, Kitzman DW, Wesley DJ, Little WC. Losartan improves exercise tolerance in patients with diastolic dysfunction and a hypertensive response to exercise. J Am Coll Cardiol. 1999;33(6):1567-72.

24 Hamroff G, Katz SD, Mancini D, Blaufarb I, Bijou R, Patel R, et al. Addition of angiotensin II receptor blockade to maximal angiotensinconverting enzyme inhibition improves exercise capacity in patients with severe congestive heart failure. Circulation. 1999;99(8):990-2.

25 Zankl AR, Ivandic B, Andrassy M, Volz HC, Krumsdorf U, Blessing E, et al. Telmisartan improves absolute walking distance and endothelial function in patients with peripheral artery disease. Clin Res Cardiol. 2010;99(12): 787-94.

26 Neumann J, Ligtenberg G, Klein IH, Boer P, Oey PL, Koomans HA, et al. Sympathetic hyperactivity in hypertensive chronic kidney disease patients is reduced during standard treatment. Hypertension. 2007;49(3):506-10.

27 Nap A, Balt JC, Mathy MJ, Van Zwieten PA. AT(1)-receptor blockade and sympathetic neurotransmission in cardiovascular disease. Auton Autacoid Pharmacol. 2003;23(5-6): 285-96.

28 Diz DI, Arnold AC, Nautiyal M, Isa K, Shaltout HA, Tallant EA. Angiotensin peptides and central autonomic regulation. Curr Opin Pharmacol. 2011;11(2):131-7.
29 Luscher TF. Endothelial dysfunction: the role and impact of the renin-angiotensin system. Heart. 2000;84(Suppl 1):i20-2; discussion i50.

30 Charkoudian N, Joyner MJ, Barnes SA, Johnson CP, Eisenach JH, Dietz NM, et al. Relationship between muscle sympathetic nerve activity and systemic hemodynamics during nitric oxide synthase inhibition in humans. Am J Physiol Heart Circ Physiol. 2006;291(3): H1378-83.

31 Ligtenberg G, Blankestijn PJ, Oey PL, Klein IH, Dijkhorst-Oei LT, Boomsma F, et al. Reduction of sympathetic hyperactivity by enalapril in patients with chronic renal failure. $\mathrm{N}$ Engl J Med. 1999;340(17):1321-8.

32 Li S, Wu Y, Yu G, Xia Q, Xu Y. Angiotensin II receptor blockers improve peripheral endothelial function: a meta-analysis of randomized controlled trials. PLoS One. 2014;9(3): e90217.

33 Shahin Y, Khan JA, Samuel N, Chetter I. Angiotensin converting enzyme inhibitors effect on endothelial dysfunction: a meta-analysis of randomised controlled trials. Atherosclerosis. 2011;216(1):7-16.

34 Levey AS, Coresh J, Greene T, Stevens LA, Zhang YL, Hendriksen S, et al. Using standardized serum creatinine values in the modification of diet in renal disease study equation for estimating glomerular filtration rate. Ann Intern Med. 2006;145(4):247-54

35 Whelton PK, Carey RM, Aronow WS, Casey DE Jr, Collins KJ, Dennison Himmelfarb C, et al. 2017 ACC/AHA/AAPA/ABC/ACPM/ AGS/APhA/ASH/ASPC/NMA/PCNA guideline for the prevention, detection, evaluation, and management of high blood pressure in adults: a report of the American College of Cardiology/American Heart Association Task Force on clinical practice guidelines. J Am Coll Cardiol. 2018;71(19):e127-248.

36 Wallin BG, Fagius J. Peripheral sympathetic neural activity in conscious humans. Annu Rev Physiol. 1988;50:565-76.

37 Jeleazcov C, Krajinovic L, Münster T, Birkholz T, Fried R, Schüttler J, et al. Precision and accuracy of a new device (CNAPTM) for continuous non-invasive arterial pressure monitoring: assessment during general anaesthesia. Br J Anaesth. 2010;105(3):264-72.

38 Mano T, Iwase S, Toma S. Microneurography as a tool in clinical neurophysiology to investigate peripheral neural traffic in humans. Clin Neurophysiol. 2006;117(11):2357-84.

39 Park J, Liao P, Sher S, Lyles RH, Deveaux DD, Quyyumi AA. Tetrahydrobiopterin lowers muscle sympathetic nerve activity and improves augmentation index in patients with chronic kidney disease. Am J Physiol Regul Integr Comp Physiol. 2015;308(3):R208-18.

40 Carter CS, Onder G, Kritchevsky SB, Pahor $\mathrm{M}$. Angiotensin-converting enzyme inhibition intervention in elderly persons: effects on body composition and physical performance. J Gerontol A Biol Sci Med Sci. 2005;60(11): 1437-46.
41 Cesari M, Pedone C, Incalzi RA, Pahor M. ACE-inhibition and physical function: results from the Trial of Angiotensin-Converting Enzyme Inhibition and Novel Cardiovascular Risk Factors (TRAIN) study. J Am Med Dir Assoc. 2010;11(1):26-32.

42 Dore A, Houde C, Chan KL, Ducharme A, Khairy P, Juneau M, et al. Angiotensin receptor blockade and exercise capacity in adults with systemic right ventricles: a multicenter, randomized, placebo-controlled clinical trial. Circulation. 2005;112(16):2411-6.

43 Gerdts E, Björnstad H, Devereux RB, LundJhansen P, Davidsen ES, Omvik P. Exercise performance during losartan- or atenololbased treatment in hypertensive patients with electrocardiographic left ventricular hypertrophy (a LIFE substudy). Blood Press. 2006; 15(4):220-6.

44 Gray SL, LaCroix AZ, Aragaki AK, McDermott M, Cochrane BB, Kooperberg CL, et al. Angiotensin-converting enzyme inhibitor use and incident frailty in women aged 65 and older: prospective findings from the Women's Health Initiative Observational Study. J Am Geriatr Soc. 2009;57(2):297-303.

45 Leon SJ, Tangri N. The use of renin-angiotensin system inhibitors in patients with chronic kidney disease. Can J Cardiol. 2019;35(9): 1220-7.

46 Rajagopalan S, Kurz S, Münzel T, Tarpey M, Freeman BA, Griendling KK, et al. Angiotensin II-mediated hypertension in the rat increases vascular superoxide production via membrane NADH/NADPH oxidase activation. Contribution to alterations of vasomotor tone. J Clin Invest. 1996;97(8):1916-23.

47 Henriksen EJ, Jacob S. Modulation of metabolic control by angiotensin converting enzyme (ACE) inhibition. J Cell Physiol. 2003; 196(1):171-9.

48 Henriksen EJ, Jacob S, Kinnick TR, Youngblood EB, Schmit MB, Dietze GJ. ACE inhibition and glucose transport in insulinresistant muscle: roles of bradykinin and nitric oxide. Am J Physiol. 1999;277(1):R332-6.

49 Wray DW, Fadel PJ, Smith ML, Raven P, Sander M. Inhibition of alpha-adrenergic vasoconstriction in exercising human thigh muscles. J Physiol. 2004;555(Pt 2):545-63.

50 Vongpatanasin W, Wang Z, Arbique D, Arbique G, Adams-Huet B, Mitchell JH, et al. Functional sympatholysis is impaired in hypertensive humans. J Physiol. 2011;589(Pt 5): 1209-20.

51 Sprick JD, Downey RM, Morison DL, Fonkoue IT, Li Y, DaCosta D, et al. Functional sympatholysis is impaired in end-stage renal disease. Am J Physiol Regul Integr Comp Physiol. 2019;316(5):R504-R511.

52 Price A, Raheja P, Wang Z, Arbique D, Adams-Huet B, Mitchell JH, et al. Differential effects of nebivolol versus metoprolol on functional sympatholysis in hypertensive humans. Hypertension. 2013;61(6):1263-9. 